\title{
A Review of the Epidemiology of Temporal Lobe Epilepsy
}

\author{
Jose F. Téllez-Zenteno and Lizbeth Hernández-Ronquillo \\ Division of Neurology, Department of Medicine, Royal University Hospital, University of Saskatchewan, Saskatoon, \\ SK, Canada S7N OW8
}

Correspondence should be addressed to Jose F. Téllez-Zenteno, jftellez@yahoo.com

Received 22 July 2011; Revised 6 October 2011; Accepted 5 November 2011

Academic Editor: Seyed M. Mirsattari

Copyright ( $) 2012$ J. F. Téllez-Zenteno and L. Hernández-Ronquillo. This is an open access article distributed under the Creative Commons Attribution License, which permits unrestricted use, distribution, and reproduction in any medium, provided the original work is properly cited.

\begin{abstract}
Partial-onset epilepsies account for about $60 \%$ of all adult epilepsy cases, and temporal lobe epilepsy (TLE) is the most common type of partial epilepsy referred for epilepsy surgery and often refractory to antiepileptic drugs (AEDs). Little is known about the epidemiology of TLE, because it requires advanced neuroimaging, positive EEG, and appropriate clinical semiology to confirm the diagnosis. Moreover, recently recognized incidentally detected mesial temporal sclerosis in otherwise healthy individuals and benign temporal epilepsy indicate that the true epidemiology of TLE is underestimated. Our current knowledge on the epidemiology of TLE derives from data published from tertiary referral centers and/or inferred from population-based studies dealing with epilepsy. This article reviews the following aspects of the epidemiology of TLE: definitions, studies describing epidemiological rates, methodological observations, the interpretation of available studies, and recommendations for future studies.
\end{abstract}

\section{Introduction}

Epidemiology is the study of disease frequency, its determinants, natural history, and burden of illness in populations [1]. There are many shortcomings in the understanding of the epidemiology of epilepsy mainly related to methodological problems. Some of them are diagnostic accuracy, case ascertainment, and selection bias [2]. Information regarding the epidemiology of temporal lobe epilepsy (TLE) is scanty with few studies and review articles in this subject [3]. This article reviews some of the published evidence in the area and analyzes the methodological obstacles to perform epidemiological studies in this type of epilepsy. This article explores the following aspects; a review of the available definitions for TLE, measures in epidemiology, and a brief review of the well-knwon epidemiological rates in epilepsy. The article finishes with the description and analysis of the available epidemiological studies in TLE.

\section{Definition of Temporal Lobe Epilepsy}

Standardized definitions are crucial for epidemiological research. The International League Against Epilepsy (ILAE) defines epilepsy as "a condition characterized by two or more recurrent epileptic seizures over a period longer than 24 hours, unprovoked by any immediate identified cause" $[4,5]$. This definition has been used in several epidemiological studies with great success and has helped to understand the burden of epilepsy in different countries [6-8]. A modification in the definition was suggested by Fisher et al. [9] in 2005. The new definition requires the occurrence of at least one seizure plus a clear predisposing factor [9]. This modification could have practical consequences because the prevalence of epilepsy can be overestimated.

TLE was included in the classification of the ILAE in 1989 under the group of localization-related symptomatic epilepsies. The definition offers a tentative description based on suggestive clinical features (dividing seizures in simple and complex partial seizures) plus ictal and interictal electroencephalogram (EEG) findings [10]. The definition suggests the use of some findings in additional tests to help in the diagnosis, such as the presence of hypometabolism in proton-emission tomography scan [10]. The classification of the ILAE also identifies seizures coming from the amygdalohippocampal area (mesiobasal limbic or rhinencephalic) and seizures coming from the lateral temporal area [10]. The latter is a form of TLE which is often referred to as neocortical TLE (nTLE). 
The classification released by the ILAE in 2010 recognizes some diagnostically meaningful forms of epilepsy such as mesial temporal lobe epilepsy (mTLE) with hippocampal sclerosis (HS) that was not included in the previous classifications [11]. mTLE was defined as a symptomatic focal epilepsy that can be subcategorized as limbic epilepsy (versus neocortical epilepsy) [10] and is one of the most common types of epilepsy referred for epilepsy surgery, often refractory to antiepileptic drugs (AEDs). This classification also suggests the removal of the term "symptomatic" and uses the term "focal." It also adds the terms genetic, structural, and unknown etiology which may help to create broad categories to better classify some patients. Finally, as Wiebe [3] pointed out, the previous and current classifications of the ILAE do not rate the diagnosis of TLE and the other types of seizures or syndromes according to the diagnostic certainty, for example, definite, probable, and possible. Also, there is no weight for diagnostic features specifying major or minor criteria.

In general, the positive points of the previous and current classifications are as follows: the diagnosis is based on typical clinical description, there is recognition of anatomical areas, the addition of mTLE with HS which is a common type of syndrome, and the inclusion of some specific features such as genetic, structural, and unknown that may help to classify some etiologies in patients with TLE. The negative aspects of the available classifications are the exclusion of MRI criteria and video-EEG findings to diagnose and classify patients, especially for patients with no findings in the routine EEG despite a potential clinical description of TLE. The current classifications also do not include diagnostic categories such as definite, probable, and possible. The latter aspect is a significant disadvantage for the development of large epidemiological studies in TLE.

\section{The Main Measures in Epidemiology of Epilepsy}

In order to perform epidemiological studies in epilepsy, the ILAE recommends the following measures [3]. (a) Point prevalence is the total number of patients with epilepsy in a given population at a specified point in time. (b) Period prevalence is the number of patients with epilepsy in a given population during a defined time interval. (c) Lifetime prevalence is the number of patients with a history of epilepsy, regardless of treatment or recent seizure activity. (d) Incidence is a measure of the risk of developing some new condition within a specified period of time. (e) Incidence proportion (also known as cumulative incidence) is the number of new cases within a specified time period divided by the size of the population initially at risk. (f) The standard mortality ratio (SMR) is the ratio of observed deaths to expected deaths in a defined population. It is age- and gender-adjusted to a standard population and is the most widely used measure of mortality.

\section{Epidemiology of Epilepsy}

4.1. Prevalence. The prevalence of epilepsy in the developed countries ranges from 4 to 10 cases per 1,000 [1]. Studies in the developing and tropical countries have reported a higher prevalence rates of epilepsy, ranging from 14 to 57 cases per 1,000 persons $[12,13]$. Higher prevalence rates of epilepsy in the developing countries is probably related to the methodological aspects of those studies, although in some regions in the world, specific infectious diseases are frequent causes of epilepsy such as neurocysticercosis [13].

4.2. Incidence. The median incidence rate of epilepsy in the developed countries ranges from 25-50 per 100,000 person years while in the developing countries it ranges from approximately $30-115$ per 100,000 person years [14]. In the developed countries, the incidence of epilepsy tends to exhibit a U-shaped curve with highest rates in the children and the elderly. This same pattern has not been found in the developing countries, where the incidence of epilepsy appears to peak in early adulthood.

4.3. Mortality. SMR in epilepsy ranges from 1.2 and 9.3 and depends on study methods and population [15-17]. Overall, the information that proves that mortality is increased in patients with epilepsy versus different type of controls is very solid and comes from well-designed controlled studies [1517].

\section{Epidemiology of Temporal Epilepsy}

Unfortunately, there are few epidemiological studies in TLE. The majority of the studies have been generated in referral centers providing biased estimates.

The best available epidemiological data was provided by the Hauser and Kurland [18]. This initial epidemiological study explored different epidemiological markers of epilepsy in the community of Rochester Minnesota from 1935 to 1967. All the cases were assessed by experts in the Mayo Clinic which was the sole provider of neurology service including EEG to that community. The incidence rate of TLE was 10.4 per 100,000 between 1945 and 1964 and 6.5 between 1935 and 1944. In the same periods of time, corresponding incidence rates of epilepsy in the whole population were 54.3 and 34.7, respectively. In the same study, the calculated prevalence of TLE in 1960 was 1.7 per 1,000 people, with a corresponding rate of epilepsy in the whole population of 6.2 cases. In further studies published by Hauser et al. [19, 20], in the same community, estimates of prevalence and incidence of partial epilepsy were reported, but not for TLE.

Other estimates regarding the prevalence of epilepsy have been obtained from tertiary referral centers. Semah et al. [21] published a very important study where 2,200 patients with epilepsy attending a tertiary care center were classified according the criteria of the ILAE. In this study, 1369 patients $(62.2 \%)$ had localization-related epilepsy. From these cases, $66 \%$ had TLE, $24 \%$ of the cases frontal epilepsy, $2 \%$ parietal, 3\% occipital, and 3\% multilobar. From the whole cohort, $24 \%$ had temporal epilepsy. The rest of the patients had either generalized epilepsy $(21.5 \%)$ or undetermined whether partial or generalized (16.3\%). The study also compares rates of intractability between partial and generalized epilepsy being the first one more intractable. 
Semah et al. [21] stated the difficulties to localize the epileptogenic zones in some patients and the classification was difficult to use.

A study of Manford et al. [22] produced estimates from a different source. In this study, 275 general practitioners identified all patients older than one month in whom a new diagnosis of definite or possible epileptic seizures was made during 3 years of recruitment. The practices of those family physicians were located around the country in urban and rural areas to avoid demographic sources of bias. Patients were followed at six months, and then every year with followup between 4 and 7 years. Details of hospital and specialist assessments and results of investigations were obtained. From 1995 patients that were included in that cohort, only $594(21 \%)$ patients were classified as having definite epileptic seizures. The authors had significant problems to identify the localization of the epileptogenic area as many patients did not have video-EEG telemetry. Two hundred and forty five (41\%) patients were classified as having localization-related epilepsy. Only 3 cases were classified as clear nTLE, 9 overlap between TLE and frontal lobe epilepsy (FLE), and 40 cases were diagnosed as TLE but the researchers could not distinguish between nLTE and mLTE. The overall percentage of cases with TLE from the group of focal cases was $21 \%$ and from the whole cohort was $9 \%$.

Finally, studies from surgical centers describe the frequency of TLE and report different rates compared with other sources. For example, Wass et al. [23] reported 291 patients who had epilepsy surgery, 73\% were TLE cases and $27 \%$ extratemporal. Guldvog et al. [24] described the seizure outcomes of 64 patients, 34 (53\%) had TLE and $30(47 \%)$ in other locations. Keene et al. [25] reported 64 patients, $44(69 \%)$ were temporal resections, $16(25 \%)$ extratemporal, and $4(6 \%)$ hemispherectomies. Rougier et al. [26] reported 100 resections, $76 \%$ were temporal, $23 \%$ frontal and 1\% parietal. Finally, Daniel and Chandy [27] reported the surgical outcomes of 141 patients, 102 (73\%) patients had temporal resections and 39 (27\%) had extratemporal resections. All the studies in surgical centers provide similar rates which are significantly higher than other sources.

Regarding mortality which is a very relevant outcome in epilepsy, we do not have any reported estimates in patients with TLE. There are reports from surgical centers with controversial information regarding the improvement of mortality rates in TLE after surgery. Some studies have demonstrated an improvement of mortality rates after epilepsy surgery if patients render seizure-free [28, 29], but these observations are not consistent in all studies $[9,30]$. Patients with TLE from epilepsy centers represent very selected populations and they do not accurately reflect mortality in epilepsy in TLE.

\section{Interpretation of the Published Epidemiological Studies}

The available studies describing the epidemiology of TLE provide interesting and varied information. The studies by
Manford et al. $[22,31]$ provide a unique epidemiological view of TLE. In this study, the patients did not belong to surgical centers or epilepsy centers and were mainly recruited by general practitioners. The overall percentage of patients with TLE from the groups of patients with localizationrelated epilepsy was $21 \%$ and from the whole cohort was $9 \%$. This study clearly indicates that TLE is not the most frequent type of epilepsy if we take into account cases with epilepsy from the community.

On the other hand, there is no doubt that TLE is the most frequent type of epilepsy that is referred to the surgical centers. The majority of surgical series report that TLE represents between 50 and $73 \%$ of all cases assessed in epilepsy surgical centers. The high prevalence of patients with TLE in surgical centers is probably related to the better surgical outcome of TLE compared to extratemporal epilepsy and the higher risk of neurological deficits related to excision of functional cortex in extratemporal cases. Because of this reason, more neurologists and more family practitioners possibly refer patients with TLE more frequently for surgical assessment. In addition, some studies have demonstrated that TLE has high rates of intractability and it could be another reason why patients are more referred to epilepsy centers.

The intermediate rates of TLE reported in tertiary referral centers help us to understand the epidemiology of this entity. Semah et al. [21] showed in his study that overall $24 \%$ of patients referred to tertiary care centers had TLE. From the group of patients with localization-related epilepsies, TLE was the most frequent with $66 \%$ of cases. In contrast to the studies from epilepsy surgical centers where a great majority of cases have TLE and from studies based on community referrals where the rate is low, studies from tertiary centers show an intermediate prevalence of TLE.

The study of Hauser and Kurland [18] is unique in many aspects and the methodology that was used in this study is probably the recommended for a large-scale epidemiological study in TLE. In the study of Hauser and Kurland [18], all the patients were evaluated in the Mayo Clinic, allowing the possibility to have the assessment from the specialist and the use of other tests to classify the patients with the different types of epilepsy. In contrast to many epidemiological studies that asses the overall prevalence of epilepsy and where simple methods can be used such as a questionnaire or simple questions to ascertain the diagnosis of epilepsy to obtain accurate estimates, in the specific case of TLE and other partial epilepsies, it would be necessary to have a clinical assessment by specialists and the use of other tests such as EEG, MRI, and in some cases video-EEG telemetry.

\section{Future Research}

The lack of large-scale epidemiological studies in TLE could be related to real methodological obstacles. Probably the main obstacle is the lack of a clear definition. As was reviewed in this article, the current definition by the ILAE is not easy to apply in large epidemiological studies. Examples of this situation are the studies of Manford et al. and Semah et al. [21, 31], where some patients were not accurately 
classified because of the limitations of the definitions already discussed. In the future, a definition that could include a clinical description plus EEG, MRI, and video-EEG criteria should be employed to improve the classification of patients with TLE. Moreover, the definition has to be easy to apply in large populations such as the ILAE definition of epilepsy.

In the earlier epidemiological studies, very little if any neuroimaging was done; it was not yet available. With the expanded use of neuroimaging in the diagnostic evaluation of most patients with seizures and the improvement in imaging technology, we are getting a better understanding of the role of structural lesions in patients with TLE. Few studies have explored the presence of specific imaging findings related with TLE, such as MTS in normal population or in patients with benign forms of TLE $[32,33]$. In the future, epidemiological studies using MRI are going to be critical in order to understand better the epidemiology of TLE.

Another challenge for future epidemiological studies in TLE is the selection of the population. As we reviewed in this article, a cohort for epidemiological studies on TLE should be based on a community where a hospital with major resources to investigate patients is available. This is necessary considering that many cases cannot be diagnosed only with the clinical history and they will require other specialized tests.

Finally, a significant problem to develop a large epidemiological study is the low incidence and prevalence of TLE. The study of Hauser and Kurland [18] shows that the rates are low and the necessity of a large sample size for epidemiological studies could be not feasible in many settings.

\section{Acknowledgment}

J. F. Tellez-Zenteno receives grants from the University of Saskatchewan and the Royal University Hospital Foundation, Saskatoon, Saskatchewan, through the Mudjadik Thyssen Mining Professorship in Neurosciences.

\section{References}

[1] G. S. Bell and J. W. Sander, "CPD-Education and selfassessment. The epidemiology of epilepsy: the size of the problem," Seizure, vol. 10, no. 4, pp. 306-316, 2001.

[2] A. T. Berg, F. M. Testa, S. R. Levy, and S. Shinnar, "The epidemiology of epilepsy: past, present, and future," Neurologic Clinics, vol. 14, no. 2, pp. 383-398, 1996.

[3] S. Wiebe, "Epidemiology of temporal lobe epilepsy," Canadian Journal of Neurological Sciences, vol. 27, supplement 1, pp. S6S10, 2000.

[4] J. W. A. S. Sander, "The epidemiology of the epilepsies: future directions," Epilepsia, vol. 38, no. 5, pp. 614-618, 1997.

[5] Guidelines for Epidemiologic Studies on Epilepsy, "Commission on epidemiology and prognosis, international league against epilepsy," Epilepsia, vol. 34, no. 4, pp. 592-596, 1993.

[6] A. Õun, S. Haldre, and M. Mägi, "Prevalence of adult epilepsy in Estonia," Epilepsy Research, vol. 52, no. 3, pp. 233-242, 2003.

[7] W. Dent, R. Helbok, W. B. P. Matuja, S. Scheunemann, and E. Schmutzhard, "Prevalence of active epilepsy in a rural area in south Tanzania: a door-to-door survey," Epilepsia, vol. 46, no. 12, pp. 1963-1969, 2005.
[8] M. C. Picot, M. Baldy-Moulinier, J. P. Daurès, P. Dujols, and A. Crespel, "The prevalence of epilepsy and pharmacoresistant epilepsy in adults: a population-based study in a Western European country," Epilepsia, vol. 49, no. 7, pp. 1230-1238, 2008.

[9] R. S. Fisher, W. van Emde Boas, W. Blume et al., "Epileptic seizures and epilepsy: definitions proposed by the International League Against Epilepsy (ILAE) and the International Bureau for Epilepsy (IBE)," Epilepsia, vol. 46, no. 4, pp. 470472, 2005.

[10] J. Roger, F. E. Dreifuss, M. Martinez-Lage et al., "Proposal for revised classification of epilepsies and epileptic syndromes," Epilepsia, vol. 30, no. 4, pp. 389-399, 1989.

[11] A. T. Berg, S. F. Berkovic, M. J. Brodie et al., "Revised terminology and concepts for organization of seizures and epilepsies: report of the ILAE Commission on Classification and Terminology, 2005-2009," Epilepsia, vol. 51, no. 4, pp. 676-685, 2010.

[12] J. G. Burneo, J. Tellez-Zenteno, and S. Wiebe, "Understanding the burden of epilepsy in Latin America: a systematic review of its prevalence and incidence," Epilepsy Research, vol. 66, no. 1-3, pp. 63-74, 2005.

[13] A. Carpio and W. A. Hauser, "Epilepsy in the developing world," Current Neurology and Neuroscience Reports, vol. 9, no. 4, pp. 319-326, 2009.

[14] I. A. W. Kotsopoulos, T. van Merode, F. G. H. Kessels, M. C. T. F. M. De Krom, and J. A. Knottnerus, "Systematic review and meta-analysis of incidence studies of epilepsy and unprovoked seizures," Epilepsia, vol. 43, no. 11, pp. 1402-1409, 2002.

[15] A. Berg, "Mortality in epilepsy," Epilepsy Currents, vol. 1, no. 1, article 28, 2001.

[16] N. Hitiris, R. Mohanraj, J. Norrie, and M. J. Brodie, "Mortality in epilepsy," Epilepsy and Behavior, vol. 10, no. 3, pp. 363-376, 2007.

[17] T. Tomson, "Mortality in epilepsy," Journal of Neurology, vol. 247, no. 1, pp. 15-21, 2000.

[18] W. A. Hauser and L. T. Kurland, "The epidemiology of epilepsy in Rochester, Minnesota, 1935 through 1967," Epilepsia, vol. 16, no. 1, pp. 1-66, 1975.

[19] W. A. Hauser, J. F. Annegers, and L. T. Kurland, "Prevalence of epilepsy in Rochester, Minnesota: 1940-1980," Epilepsia, vol. 32, no. 4, pp. 429-445, 1991.

[20] W. A. Hauser, J. F. Annegers, and L. T. Kurland, "Incidence of epilepsy and unprovoked seizures in Rochester, Minnesota: 1935-1984," Epilepsia, vol. 34, no. 3, pp. 453-468, 1993.

[21] F. Semah, M. C. Picot, C. Adam et al., "Is the underlying cause of epilepsy a major prognostic factor for recurrence?" Neurology, vol. 51, no. 5, pp. 1256-1262, 1998.

[22] M. Manford, Y. M. Hart, J. W. A. S. Sander, and S. D. Shorvon, "The National General Practice Study of Epilepsy: the syndromic classification of the International League Against Epilepsy applied to epilepsy in a general population," Archives of Neurology, vol. 49, no. 8, pp. 801-808, 1992.

[23] C. T. Wass, M. M. Rajala, J. M. Hughes, and F. W. Sharbrough, "Long-term follow-up of patients treated surgically for medically intractable epilepsy: results in 291 patients treated at mayo clinic rochester between july 1972 and march 1985," Mayo Clinic Proceedings, vol. 71, no. 11, pp. 1105-1113, 1996.

[24] B. Guldvog, Y. Loyning, E. Hauglie-Hanssen, S. Flood, and H. Bjornaes, "Surgical treatment for partial epilepsy among Norwegian children and adolescents," Epilepsia, vol. 35, no. 3, pp. 554-565, 1994.

[25] D. L. Keene, M. J. Higgins, and E. C. G. Ventureyra, "Outcome and life prospects after surgical management of medically 
intractable epilepsy in patients under 18 years of age," Child's Nervous System, vol. 13, no. 10, pp. 530-535, 1997.

[26] A. Rougier, J. F. Dartigues, D. Commenges, B. Claverie, P. Loiseau, and F. Cohadon, "A longitudinal assessment of seizure outcome and overall benefit from 100 cortectomies for epilepsy," Journal of Neurology Neurosurgery and Psychiatry, vol. 55, no. 9, pp. 762-767, 1992.

[27] R. T. Daniel and M. J. Chandy, "Epilepsy surgery : overview of forty years experience," Neurology India, vol. 47, no. 2, pp. 98-103, 1999.

[28] M. J. Hennessy, Y. Langan, R. D. C. Elwes, C. D. Binnie, C. E. Polkey, and L. Nashef, "A study of mortality after temporal lobe epilepsy surgery," Neurology, vol. 53, no. 6, pp. 12761283, 1999.

[29] M. R. Sperling, A. Harris, M. Nei, J. D. Liporace, and M. J. O'Connor, "Mortality after epilepsy surgery," Epilepsia, vol. 46, no. 11, pp. 49-53, 2005.

[30] J. F. Téllez-Zenteno, R. Dhar, L. Hernandez-Ronquillo, and S. Wiebe, "Long-term outcomes in epilepsy surgery: antiepileptic drugs, mortality, cognitive and psychosocial aspects," Brain, vol. 130, no. 2, pp. 334-345, 2007.

[31] M. Manford, Y. M. Hart, J. W. A. S. Sander, and S. D. Shorvon, "National General Practice Study of Epilepsy (NGPSE): partial seizure patterns in a general population," Neurology, vol. 42, no. 10, pp. 1911-1917, 1992.

[32] S. R. Benbadis, J. Wallace, F. Reed Murtagh, C. Martinez, W. O. Tatum, and F. L. Vale, "MRI evidence of mesial temporal sclerosis in subjects without seizures," Seizure, vol. 11, no. 5, pp. 340-343, 2002.

[33] A. Labate, P. Ventura, A. Gambardella et al., "MRI evidence of mesial temporal sclerosis in sporadic "benign" temporal lobe epilepsy," Neurology, vol. 66, no. 4, pp. 562-565, 2006. 


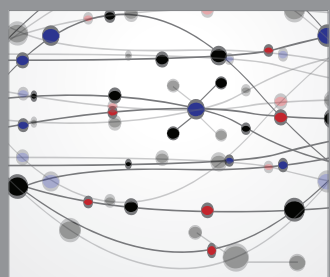

The Scientific World Journal
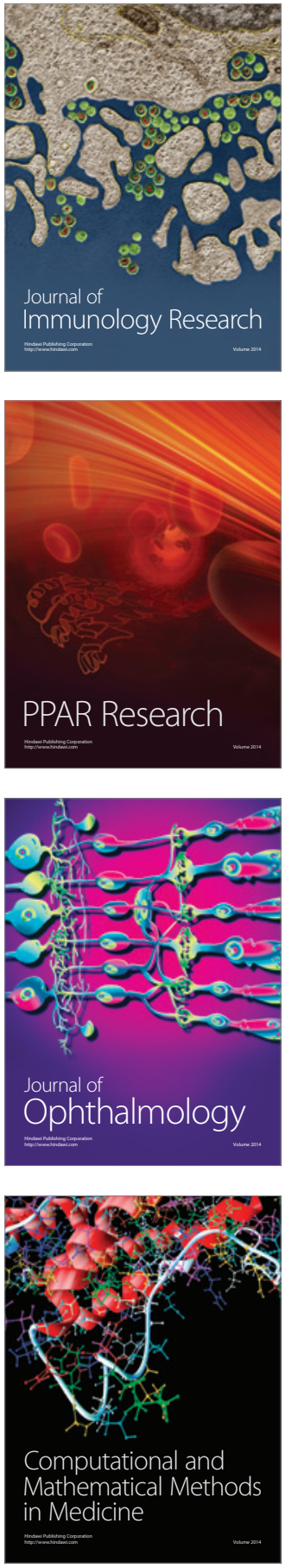

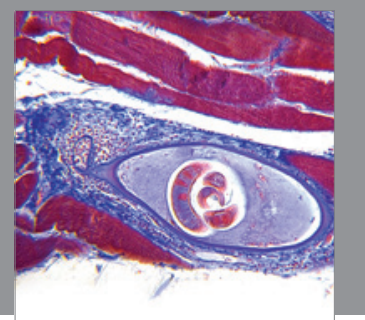

Gastroenterology

Research and Practice
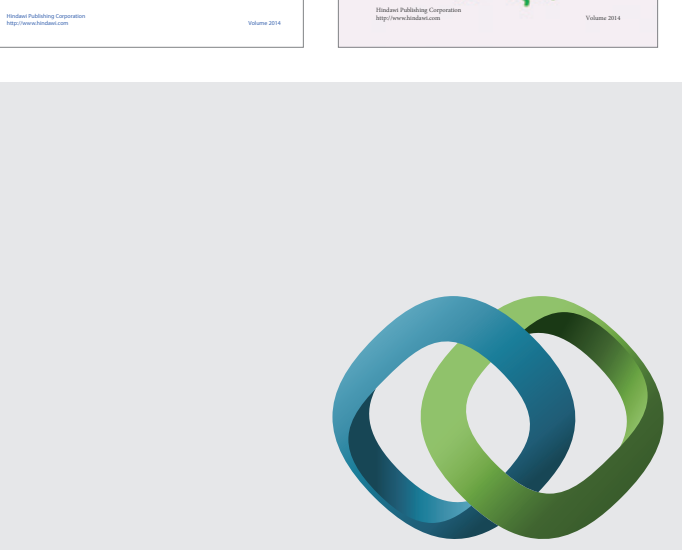

\section{Hindawi}

Submit your manuscripts at

http://www.hindawi.com
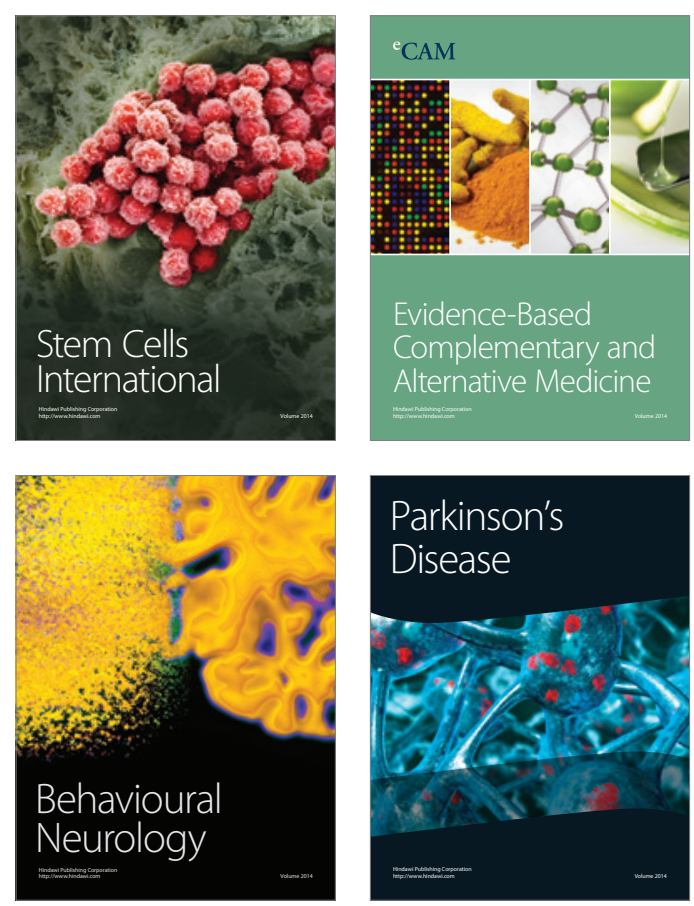

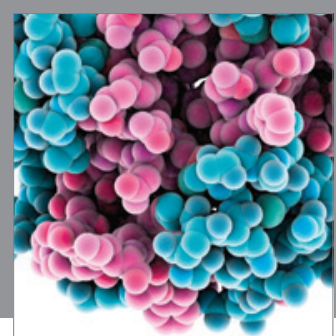

Journal of
Diabetes Research

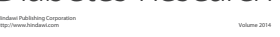

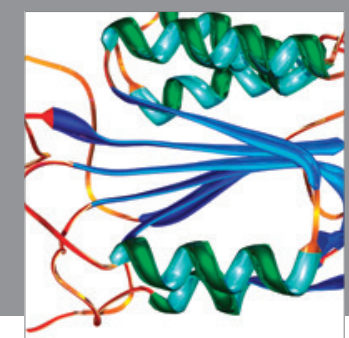

Disease Markers
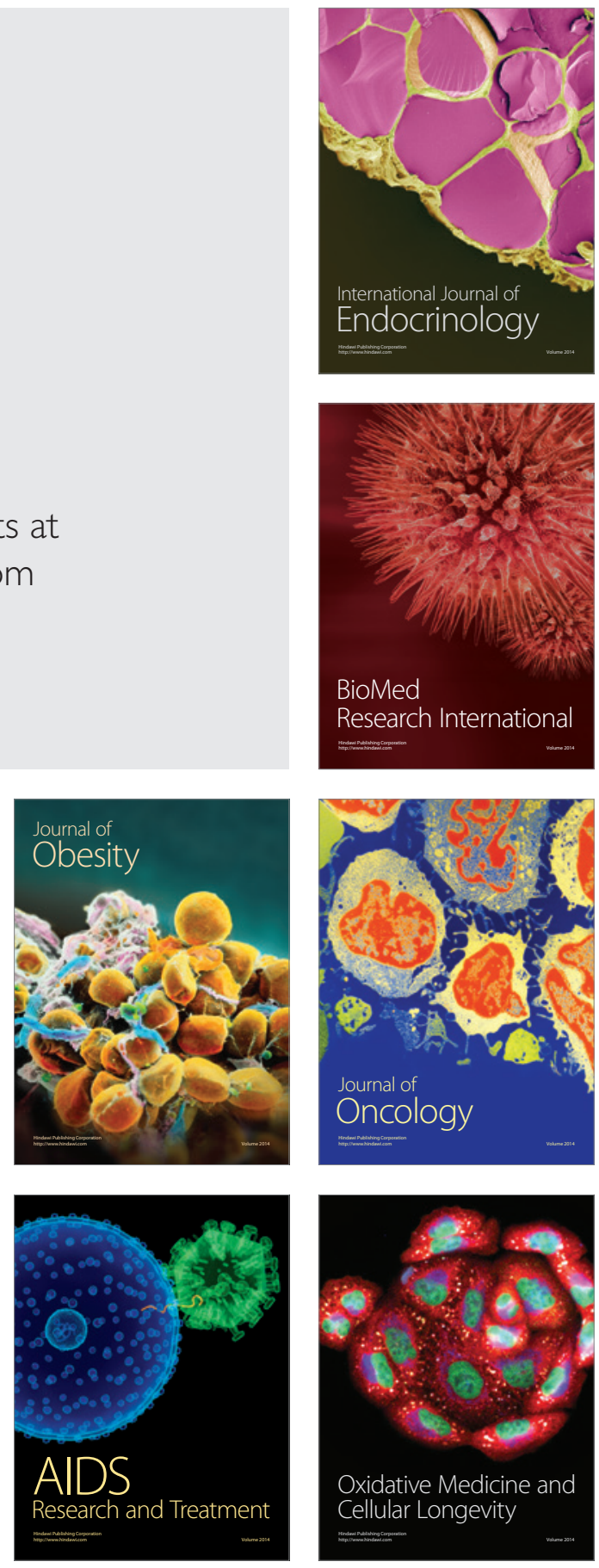